\title{
Conservative approach to the mandible for a resection of a massive florid cemento-osseous dysplasia, Case report
}

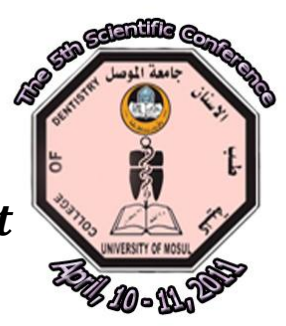

\begin{abstract}
All variations of fibro-osseous diseases demonstrate replacement of normal bone by fibrous connective tissue matrix within which varying amounts or combinations of osteoid and mature bone and in some instances cementum-like tissue are deposited. Case Report: Patient aged 46 years, was blind since 1991, admitted to the Department of oral and maxillofacial surgery, (Alsalam Teaching Hospital, Mosul), June 2010, presented with a 14 years duration increasingly bilateral massive hard swelling in the lower jaw. The decision taken according to the clinical and radiographical findings, that matching the presentation of florid cemento-osseous dysplasia, the affected mandible resected through a conservative 3 incisions. Results: the $\mathrm{H}$ and $\mathrm{E}$ stain examination of the whole resected mass reveals a definite diagnosis of florid cemento-osseous dysplasia. Discussion: Benign gnathic lesions, even massive, could be resected via a conservative surgical approaches, the histopathological findings is a type of investigation and not the only sole clue, while the sum of clinical findings and other aids of investigations make the final decision.
\end{abstract}

${ }^{1}$ Bashar A Tawfeeq (FICMS, Maxfacs); Rawaa Y. Khaleel (MSC, MOMS.RCPSG); Bashar

A. Hassawi $(M B C H B, M I B M S)$;

Aws Gh. Younis (BDS.SHO)

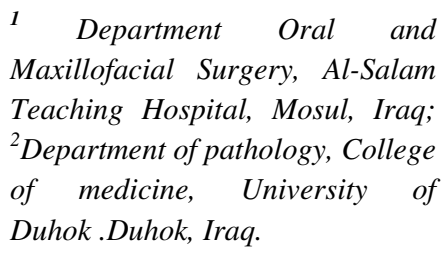

Key Words: mandible resection and florid cemento-osseous dysplasia

\section{INTRODUCTION}

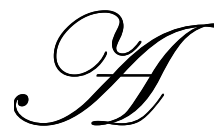

ll variations of fibro-osseous diseases demonstrate replacement of normal bone by fibrous connective tissue matrix within which varying amounts or combinations of osteoid and mature bone and in some instances cementum-like tissue are deposited ${ }^{1}$.

Any discussion of fibro-osseous disease must begin with the observation that the term fibro-osseous is largely descriptive, nosologically limited, and diagnostically non-specific. Oral pathologists generally agree that categorical separation of the fibro-osseous diseases purely on the basis of microscopic features is, if not impossible, certainly difficult, given the enormous degree of histologic overlap that characterizes these diverse conditions. ${ }^{2}$

Bone cell functions during skeletal developments and maintenance are regulated by an array of systemic and local factors within the skeletal microenvironments. Many of these are produced by cells other than bone cell that reside in soft tissues associated with bone, such as periosteal connective tissue or bone marrow compartment. ${ }^{3}$ 
Classification: Waldron, 1993 has suggested fibro-osseous disease classification that separates lesions into three groups:

I- Fibrous dysplasia, II- Fibro-Osseous Neoplasm, III- Reactive (Dysplastic) Lesion: which include: 1-Periapical Cemento-Osseous Dysplasia, 2-Focal Cemento-Osseous Dysplasia, 3Florid Cemento-Osseous Dysplasia, 4-Familial Gigantiform Cementoma. ${ }^{2}$

* Florid Cemento-Osseous Dysplasia (FLCD):

I. Historical review and nomenclature: FLCD was initially reported as florid osseous dysplasia by Melrose et al which described a condition that has come to be accepted as the most clinically extensive form of cement-osseous dysplasia, thus the use of the term florid. ${ }^{4}$ Cases of FLCD had been published under at least 12 different names. Most prominent among these were gigantiformcementoma, chronic sclerosingosteomylities, sclerotic cemental masses, and multiple enostosis. $^{5}$

II. Clinically: According to Goaz and White 1994, Women are affected more often and the mean age of these patients is 42 years. A causal factor may be co-existent traumatic bone cyst in the molar region of the mandible. ${ }^{6}$

FLCD is frequently multi-focal and the lesions are typically more than $3 \mathrm{~cm}$. in diameter. The lesions may coalesce as the growth progresses. The most common site is the alveolar process of the jaw. Osteomylities develops in $6 \%$ of these cases. Typically, osteomylities is low grade, asymptomatic and chronic. ${ }^{7}$

Patients with FLCD may have cortical expansion, particularly of the mandible. Expansion may be pronounced enough to cause practitioners to suspect a neoplasm or Paget's disease of bone. Occasionally, patients without signs of infection complained of an intermittent, dull, aching sensation in the mandibular molar area. ${ }^{8}$

III. Radiographically, a wide spectrum is seen. Radiographs usually display diffuse distribution of lobular, irregularly shaped radiopacities throughout the alveolar process. The lobular densities are often enmeshed in poorly defined areas of decreased radiodensity, often having a ground glass appearance. ${ }^{9}$ In some instances teeth manifest hypercementosis. ${ }^{10}$ The mandibular vertical rami are spared, as is the inferior cortex. These features militate against diagnoses of fibrous dysplasia, cemento-ossifying fibroma, and to a lesser extent, Paget's disease of bone. ${ }^{11}$ Panoramic radiographs are important for accurate diagnosis. ${ }^{6}$ Patients who progress to low grade osteomyelitis demonstrate slowly progressive radiographic changes. These patients do not develop periosteal reaction or the moth-eaten appearance of typical osteomyelitis. ${ }^{10}$

IV. Management: The management consists of clinical; and radiographic observation for the life of the patient, as well as excellent periodontal and restorative care to preserve the dentition. ${ }^{12}$ The only significant complication occurs in patients who have worn tissue born prostheses for many years and who subsequently suffer severe alveolar bone resorption which serves to position the ridge mucosa between the denture base and the underlying sclerotic tissue. ${ }^{13}$ Soft tissue ulceration, exposure of the cementum like masses, and infection may result in osteomyelitis. Substantial amounts of bone can be lost as a result of these infections. ${ }^{14}$

\section{MATERIALS AND METHODS}

CASE REPORT: Patient aged 46 years, married with 6 children, from a rural area, and was blind since 1991, after a gradual declination in vision. Patient admitted to the Department of Oral and Maxillofacial Surgery in Alsalam Teaching Hospital at Mosul in June 2010, presented with a 14 years duration increasingly massive hard swelling in the lower jaw, bilaterally, with a chief complaint that a continuous facial discharge unresponsive to therapy throughout the past 10 
years. On examination, three discharging fistulae, two at the lower mandibular broad border on the left side, and the other on right side, no any sign or symptom of interrupted neural function regarding sensory or motor activity of labial and cheek musculature. Oral examination revealed a few scattered deciduous and permanent teeth throughout the upper and lower jaw, multiple protruding lower ridge masses, obliterated lower sulci and a restricted space for the tongue. Radiographical findings in panoramic and mandibular and skull postero-anterior view reveal multiple radiopaque lesions scattered throughout the mandible. A radiolucent capsule and cortical rim surrounds the lesions, and in CT scan we can show a diffuse distribution of lobular, irregularly shaped radiopacities with a ground glass appearance, both mandibular rami were unaffected together with the inferior cortex, the lesion extended from the imaginary line joining the most lower point of sigmoid notch to the posterior limit of the angle of the mandible, from one side to the other. The gnathic area is the sole affected site by the lesion in the body.

The previous multiple visits of the patient to more than one center, with the consultation to other specialities, with the confused undefinitive result of histopathological finding, result in the diagnosis of the case as a Paget's disease, and a decision to enucleate the foci of the osteomyelitis, keeping the patient on long life observation.

The decision taken in our department, according to the clinical findings, that the mass consisted of florid cemento-osseous dysplasia, in spite of histopathological report which was discarded due to unmatching to the clinical and radiographical findings, and accordingly, we decide to resect the affected mandible, even huge, in accordance to the patient's complain and his intolerance to the disastrous condition (his own words).

OPERATION: patient prepared for operation and orally intubated with difficult manipulation due to restricted airway passage, fortunately without the need for tracheostomy. Eight $\mathrm{ml}$ of 1:80.000 adrenalin containing xylocaine injected submucosally and subcutaneously surrounding the operative site, waiting $7 \mathrm{~min}$ and a bilateral, five $\mathrm{cm}$ submandibular incision on the right side, and Risdon incision on the left side, with a full length intra-oral crestal incision. The mandibular mass osteotomized in 3 lines, half centimeter posterior to the end of the mass on each side, and the third one intra-orally at the midline, the mass was sectioned to two pieces and pushed through an oral incision, a perforated $\mathrm{U}$ - shaped tray plate fixed via 2 screws on each ramal bone, and the incisions were sutured in layers and the tongue secured with a $1 \backslash 0$ nylon suture to be hold for postoperative recovery.

\section{RESULTS}

Patient still intubated with sufficient blood oxygenation (over 93\% $\mathrm{O}_{2}$ saturation), with questionable need for tracheostomy in the recovery period, the secured nylon suture removed, the patient extubated 2 hours later, hospitalized and observed meticulously for one week for any possible complications without the need for any blood transfusion, the sutures removed and the patient dismissed with an acceptable general health.

Two weeks later, the patient was able to open his mouth with an inter-labial distance 3 fingers, the plate was exposed at the anterior oral wound, and left for secondary healing, the motor nerve response functioned well, including lip and tongue movement.

The whole mass biopsied but this time in the central histopahlogical department in Duhok city, and the $\mathrm{H}$ and $\mathrm{E}$ stain examination reveals a definite diagnosis of florid cemento-osseous dysplasia. 


\section{DISCUSSIONS AND CONCLUSIONS}

Benign gnathic lesions and tumors, even massive, could be excised or resected via a conservative surgical approaches without the necessity for lip splitting, extended submandibular incision or even tracheostomy which could be carried out in many instances in the cases where the symphyseal part of the mandible is resected, for safe postoperative recovery period, in such a case, close follow up of the patient in the few postoperative hours obviate the need for tracheostomy and all its unwanted complications.

In addition, the decision regarding the final diagnosis and correct treatment plan later on, should be taken not only depending on histopathological findings because it is a type of investigation and not the only sole clue, while the sum of clinical findings and other aids of investigations make the final decision.

Finally, such a lesion, even rare, should be managed as soon as possible, in that this is the second case of the same diagnostic result that the patient being blind at their adulthood. The first case, which is not reported, was from Al-Najaf town in Iraq, and have a close clinical and radiographical presentation like this reported case. The possible explanation that the lesion may affect the optic canal leading to pressure on its contents including the optic nerve and central artery and later on a blindness, noting that the lesion also affect the maxilla but to a less extent.

\section{REFERENCES}

1- Waldron. Benign fibro-osseos-lesions of the jaws, a clinical, radiological and histologic review of 65 cases. Oral Surg Oral Med Oral Patho. 1973; 35: 340-350.

2- Waldron. Fibro-osseos-lesions of the jaws. J Oral maxillofac Surg. 1993; 51: 828-835.

3- Eisenberg E, Eisenbud L. Benign fibro-osseos-lesions: current concepts in historical perspective. Oral maxillofac Surg Clin Nor Am. 1997; 9:655-658.

4- Melrose R, Abrams A, Mills B: FLCD: Aclinico-pathological study of 34 cases. Oral Surg. Oral Med. Oral Patho.1976; 41: 62-82.

5- Weber A, Scrivani S. Mandible: anatomy, cyst, tumors and non-tumerous lesions. Head and neck imaging, st, Louis, mosby.1996, pp. 319-349.

6- Goaz P, White S: Oral radiology: Principles of interpretation. Ed. 3. St. Louis, Mosby - Year Book.1994, PP 388, 393-395, 471, 494-501.

7- Zafar A, Shafi M, Malik S. Ossifying fibroma of nasal cavity. J pma-j-pac-med-assoc.1997; Dec, 47(12); 312-3.

8- Melrose R: The clinico-pathologic spectrum of cemento-osseous dysplasia. Oral maxillofac Surg Nor Am1997; 9: 643-649.

9- Mohammadi-Araghi H: Fibro-osseous lesions of craniofacial bones: The rule of imaging. Radio Clin Nor Am 1993;31: 121-134.

10- Delbaso A: Maxillofacial imaging. Philadelphia WB. Saunders, 1990, PP. 192-193, 350, 361-368.

11- Su I, Weather Waldron Ca. Distinguishing features of focal cement-osseous dysplasia and cement-osseous fibroma, Oral Surg Oral Med Oral Patho.1997; November 84(5):540-9.

12- Schneider and Mesa. Differences between florid cement-osseous dysplasia and chronic sclerosing osteomyelitis. Oral Surg Oral Med Oral Patho. 1990; 70:308.

13- Higuchi Y, Nakamura N, Tashiro H: Clinico-pathologic study of COD producing cysts of the mandible. Oral Surg Oral Med Oral Patho.1988; 65:339-3-42.

14- Macintosh S: Juvenile fibro-csseous lesions. J Clin Pediatr Dent Winter.1996; 13(4): 121-4. 